\title{
Inhibition of calpains improves memory and synaptic transmission in a mouse model of Alzheimer disease
}

\author{
Fabrizio Trinchese, ${ }^{1}$ Mauro Fa', ${ }^{1}$ Shumin Liu, ${ }^{1}$ Hong Zhang, ${ }^{1}$ Ariel Hidalgo, ${ }^{1}$ Stephen D. Schmidt, ${ }^{2,3}$ \\ Hisako Yamaguchi, 4 Narihiko Yoshii, ${ }^{4}$ Paul M. Mathews, ${ }^{2,3}$ Ralph A. Nixon, ${ }^{2,3,5}$ and Ottavio Arancio ${ }^{1}$ \\ 1Department of Pathology and Taub Institute for Research on Alzheimer's Disease and the Aging Brain, Columbia University, New York, New York, USA. \\ 2Department of Psychiatry, New York University School of Medicine, New York, New York, USA. ${ }^{3}$ Center for Dementia Research, \\ Nathan Kline Institute for Psychiatric Research, Orangeburg, New York, USA. ${ }^{4}$ Pharmaceutical Research Division, Research Laboratory 1 (CNS), \\ Mitsubishi Pharma Corporation, Nihonbashi-Honcho, Chuo-ku, Tokyo, Japan. ${ }^{5}$ Department of Cell Biology, \\ New York University School of Medicine, New York, New York, USA.
}

\begin{abstract}
Calpains are calcium-dependent enzymes that determine the fate of proteins through regulated proteolytic activity. Calpains have been linked to the modulation of memory and are key to the pathogenesis of Alzheimer disease (AD). When abnormally activated, calpains can also initiate degradation of proteins essential for neuronal survival. Here we show that calpain inhibition through E64, a cysteine protease inhibitor, and the highly specific calpain inhibitor BDA-410 restored normal synaptic function both in hippocampal cultures and in hippocampal slices from the APP/PS1 mouse, an animal model of AD. Calpain inhibition also improved spatial-working memory and associative fear memory in APP/PS1 mice. These beneficial effects of the calpain inhibitors were associated with restoration of normal phosphorylation levels of the transcription factor CREB and involved redistribution of the synaptic protein synapsin I. Thus, calpain inhibition may prove useful in the alleviation of memory loss in $\mathrm{AD}$.
\end{abstract}

\section{Introduction}

To date, at least 14 mammalian calpains, cytosolic calcium-activated cysteine proteases, have been identified (reviewed in ref. 1). Two major forms, calpain 1 and calpain 2 , also known as $\mu$-calpain and $m$-calpain, have been linked to Alzheimer disease (AD) (2). Calpain 1, the form most concentrated in synapses (3), is abnormally activated in $\mathrm{AD}$ brain (4). Calpastatin, the endogenous inhibitor of calpains, is significantly decreased in the same neurodegenerative disorder (2). The activated form of calpain 2 is increased in neurites of neurons at risk for developing neurofibrillary pathology and is extensively bound to neurofibrillary tangles (NFTs) in brains of $\mathrm{AD}$ patients (5). Calpain overactivation triggered by abnormally high calcium levels and calpastatin depletion lead to limited cleavage or degradation of key neuronal proteins in $\mathrm{AD}$ $(2,6)$. Indeed, calpains modulate the trafficking and, indirectly, the proteolytic processing of the amyloid precursor protein (APP), a polypeptide thought to play a fundamental role in AD (7-11). Moreover, calpains influence the phosphorylation and proteolysis of tau, another protein associated with $\mathrm{AD}$ (reviewed in refs. 1, 6). Other calpain substrates affected in $\mathrm{AD}$ include CaM-kinase II $\alpha$ (CaMK-II $\alpha$ ) and PKC, 2 enzymes that regulate APP phosphorylation and influence its metabolism (12-14); second messenger-

Nonstandard abbreviations used: $A \beta$, amyloid- $\beta$ peptide; $A D$, Alzheimer disease APP, amyloid precursor protein; BDA-410, (2S)-N-(1S)-1-[(S)-Hydroxy(3-oxo-2-phenyl-1-cyclopropen-1-yl)methyl]-2-methylpropyl-2-benzenesulfonylamino-4-methylpentanamide; BST, basal synaptic transmission; CREB, cAMP regulatory elementbinding protein; E64, trans-epoxysuccinyl-L-leucylamido (4-guanidino)-butane; fEPSP, field excitatory postsynaptic potential; LTP, long-term potentiation; mEPSC, miniature excitatory postsynaptic current; pCREB, phosphorylated CREB; PS1, presenilin 1; RAWM, radial-arm water maze.

Conflict of interest: The authors have declared that no conflict of interest exists. Citation for this article: J. Clin. Invest. 118:2796-2807 (2008). doi:10.1172/JCI34254. related enzymes such as phospholipase $\mathrm{C}-1,-2,-\beta 3$ (15), and cyclindependent kinase 5 (Cdk-5) (16); transcription factors such as c-Jun, c-Fos, and IкB $(17,18)$; and the memory-related gene, cAMP regulatory element-binding protein (CREB) (19-21). Calpains also regulate cytoskeletal proteins such as spectrin (22) and MAP2 (23) and, through direct proteolytic actions and indirect modulatory effects on several protein kinases (PKC, ERK1/2, CaMK-II, and $\mathrm{Cdk}-5 / \mathrm{p} 35)$, play a key role in regulating the dynamic behavior and turnover of cytoskeletal proteins, especially those in synapses where calpain concentrations are high (3). Recently, calpain actions on the GluR1 subunit of AMPA receptors (24), amphiphysin I (25) and suprachiasmatic nucleus circadian oscillatory protein (26), have been shown to modulate synaptic activity and memory formation. Growing evidence suggests that the cognitive impairment in $\mathrm{AD}$ starts long before neuronal death and that signaling between neurons is interrupted at early stages of the disease. The importance of synaptic alterations in AD has been confirmed by studies on transgenic mouse models of $\mathrm{AD}(27)$ and on amyloid- $\beta$ peptideinduced (A $\beta$-induced) impairment of long-term potentiation (LTP), a widely studied cellular model of learning and memory (28). In this study, we examined the effect of calpain inhibition through E64, trans-epoxysuccinyl-L-leucylamido (4-guanidino)butane, and the highly specific inhibitor BDA-410, (2S)-N-(1S)1-[(S)-Hydroxy(3-oxo-2-phenyl-1-cyclopropen-1-yl)methyl]-2methylpropyl-2-benzenesulfonylamino-4-methylpentanamide, on the impairment of synaptic transmission due to overexpression of mutated forms of APP (K670N,M671L) and presenilin 1 (PS1) (M146L), each of which causes familial cases of AD. We also investigated whether calpain inhibition is beneficial against abnormal spatial-working memory and associative fear memory in transgenic mice overexpressing the 2 mutated transgenes (APP/ PS1 mice). Finally, we studied the effect of calpain inhibition on 

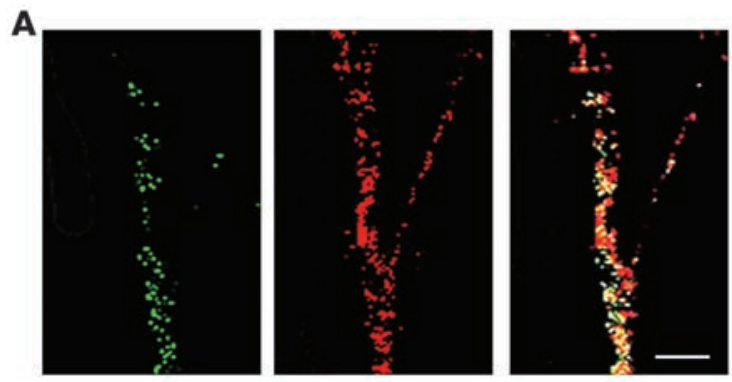

B

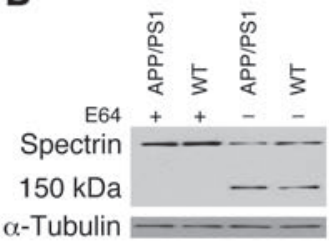

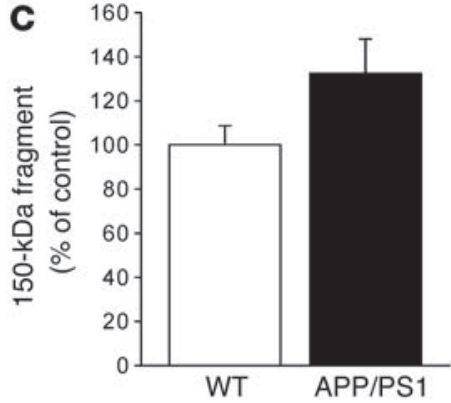

$\mathbf{E}$

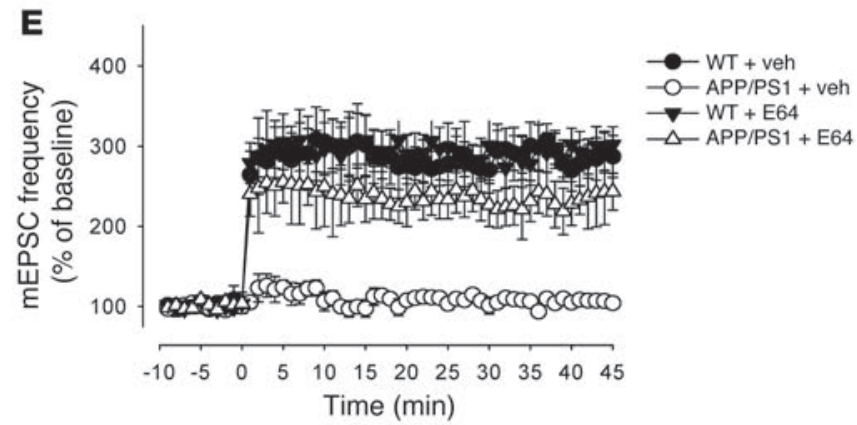

Figure 1

The calpain inhibitor E64 reestablished normal synaptic function in APP/PS1 mouse hippocampal cultures. (A) Calpain 1 immunoreactivity colocalized with immunofluorescence for the presynaptic protein, synapsin I. Calpain 1-immunoreactive puncta (left panel). Synapsin I-immunoreactive puncta (middle panel). Colocalization of calpain-immunoreactive puncta with synapsin I-immunoreactive puncta (so that the puncta appear yellow) (right panel). Scale bar: $15 \mu \mathrm{m}$. (B) Western blot demonstrated that E64 annulled calpain cleavage of spectrin to its 150-kDa fragment ( $n=5$ per group). (C) Quantitative western blot analysis of the 150-kDa fragment showed a 32\% increase in APP/PS1 cultures compared with WT cultures ( $n=5$ for both; $P<0.05$; data normalized against $\alpha$-tubulin). (D) Vehicle-treated (veh-treated) APP/PS1 cultures showed approximately 2-fold increase of spontaneous mEPSC frequency $(n=10)$ compared with vehicle-treated WT cultures $(n=10 ; P<0.01$, with 1-way ANOVA). E64 did not affect average basal mEPSC frequency in WT cultures ( $n=10 ; P>0.05$ with $t$ test) but reestablished normal basal frequency of spontaneous neurotransmitter release in APP/PS1 cultures $(n=6)$. (E) Application of glutamate no longer enhanced mEPSC frequency in cultures from vehicle-treated APP/PS1 mice compared with cultures from vehicle-treated WT mice $(n=10$ in APP/PS1 cultures; $n=10$ in WT littermate cultures; $P<0.01$ with 2-way ANOVA) without affecting mEPSC amplitude in either genotype (data not shown). Block of calpain activity through E64 ameliorated the deficit in long-lasting enhancement of synaptic transmission $(n=10 ; P<0.01$ compared with vehicle-treated APP/PS1 cultures), without affecting it in WT cultures ( $n=10 ; P>0.05$ compared with vehicle-treated WT cultures).

the phosphorylation of the memory-related molecule CREB and on the localization of the synaptic protein synapsin I.

\section{Results}

Calpain inhibition reestablishes normal synaptic function in APP/PS1 mouse cultures. Dissociated hippocampal cell cultures are a particularly useful model for examining basal neurotransmission and synaptic plasticity (29-31). Cell cultures from transgenic animals exhibit many features observed in $\mathrm{AD}$, including production of elevated levels of $A \beta$ and synaptic dysfunction $(32,33)$. Thus, as a first tool for our studies, we used hippocampal cell culture preparations derived from APP/PS1 mice (34). We first confirmed results on the synaptic localization of calpains within the hippocampal neuronal cultures (3). Calpain I immunoreactivity was present in neuronal cell bodies and was punctate along neuronal processes (Figure 1A, left panel) where it colocalized with nearly all synapsin I-immunoreactive puncta (Figure 1A, right panel, yellow). These results are consistent with the idea that both proteins are localized at the synaptic level. Regions of calpain immunoreactivity that were not associated with synapsins may represent locations where the synapse is not fully formed or areas where calpains are located extrasynaptically.

We next examined whether calpains play a role in neurotransmission using the cysteine protease inhibitor E64. E64 is an irrevers- ible, cell- and tissue-permeable inhibitor of calpain 1 and calpain 2. It is an Arg-Leu peptidomimetic with an electrophilic epoxide warhead that irreversibly modifies the active site cysteine and has been used in many studies as an inhibitor of cysteine proteases such as calpain $\left(\mathrm{IC}_{50}=0.57 \pm 0.01 \mu \mathrm{M}\right)(35-37)$. In preliminary experiments, we confirmed that E64 inhibits calpain cleavage of spectrin to its $150-\mathrm{kDa}$ fragment in neuronal cultures, a hallmark of calpain action (Figure 1B) (22). Interestingly, levels of the calpain-generated $150-\mathrm{kDa}$ fragment of spectrin were increased in APP/PS1 mouse cell cultures compared with WT cultures, suggesting that calpains are overactivated in neuronal cultures from the transgenic mice (Figure 1C).

Healthy neurons spontaneously release neurotransmitter, a phenomenon that is known as miniature excitatory postsynaptic currents (mEPSCs). The analysis of mEPSC frequency and amplitude in 11- to 14-day-old cultured neurons from WT mice did not reveal any difference among vehicle-treated cultures, those that were treated with E64 (1 $\mu \mathrm{M})$ for 3-4 days (Figure 1D), or control cultures that did not receive any treatment (Supplemental Figure 1A; supplemental material available online with this article; doi:10.1172/JCI34254DS1). These results suggest that calpains do not play a role in spontaneous release of neurotransmitter. We found a significant increase in basal mEPSC frequency 


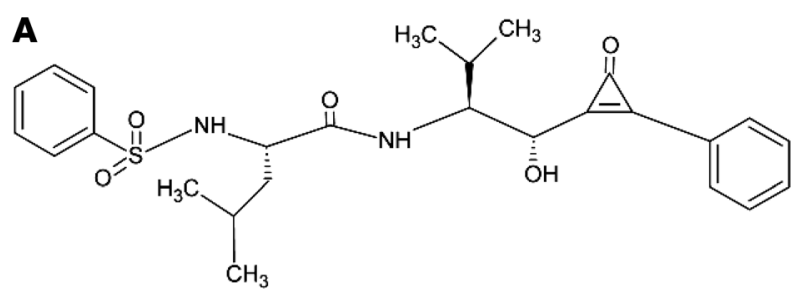

B
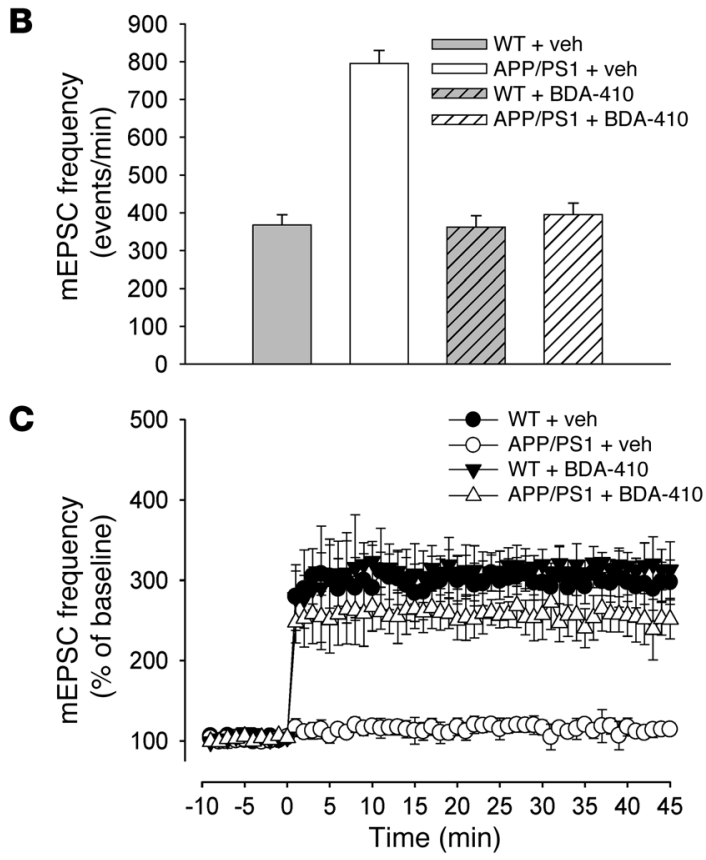

in vehicle-treated cultures from APP/PS1 animals (Figure 1D). In contrast, the average amplitude of mEPSCs was unchanged. In interleaved experiments, the application of E64 $(1 \mu \mathrm{M})$ to the culture medium for 3-4 days reestablished the normal basal frequency of spontaneous neurotransmitter release in APP/PS1 mice (Figure 1D) without changing the average amplitude. These results, which we believe to be novel, are consistent with previous findings showing that synaptic transmission is impaired following overexpression of APP and PS1 (38-40). More importantly, our findings suggest that calpain inhibitors can be used to reestablish the normal basal frequency of spontaneous release of neurotransmitter in APP/PS1 mouse cultures.

Long-lasting enhancement of synaptic transmission in the hippocampus is an important manifestation of plasticity, which is thought to underlie learning and memory (28). In preliminary experiments on hippocampal cultures from APP/PS1 mice, we found that a brief application of glutamate $(200 \mu \mathrm{M})$ did not produce the enhancement of mEPSC frequency that is seen either in cultures from WT mice treated with vehicle (Figure 1E) or in control WT cultures that did not receive any treatment (Supplemental Figure 1B). These data, which are consistent with results from slices derived from the APP/PS1 mouse (38), indicate that overexpression of these 2 transgenes inhibits the neuron's capacity for plasticity. Therefore, in interleaved experiments, we tested whether inhibiting calpain activity with 1- $\mu \mathrm{M}$ E64 makes it possible to reestablish normal synaptic plasticity in cultures from double transgenic animals. We found that APP/PS1 cultures treated with E64 (for 3-4

\section{Figure 2}

The highly selective calpain inhibitor BDA-410 reestablished normal basal frequency of spontaneous neurotransmitter release and rescued impairment of synaptic plasticity in APP/PS1 mouse cultures. (A) Chemical structure of BDA-410. (B) Vehicle-treated APP/PS1 cultures showed approximately 2-fold increase of spontaneous mEPSC frequency $(n=7)$ compared with vehicle-treated WT cultures $(n=8$; $P<0.01$ with 1 -way ANOVA). BDA-410 did not affect average basal mEPSC frequency in WT cultures $(n=7)$ and reestablished normal basal frequency of spontaneous neurotransmitter release in APP/PS1 cultures $(n=8 ; P<0.01)$. (C) Application of glutamate $(200 \mu \mathrm{M})$ did not enhance mEPSC frequency in cultures from APP/PS1 mice compared with cultures from WT mice ( $n=7$ in APP/PS1 cultures; $n=8$ in WT littermate cultures; $P<0.01$ with 2 -way ANOVA). mEPSC amplitude values were not affected by glutamate (data not shown). Block of calpain activity through BDA-410 was beneficial against the impairment of long-lasting enhancement of synaptic transmission $(n=8 ; P<0.01$ compared with vehicle-treated APP/PS1 cultures), without affecting it in WT cultures ( $P>0.05$ compared with vehicle-treated WT cultures).

days prior to recording) showed normal long-lasting enhancement of synaptic plasticity (Figure 1E). Moreover, E64-treated WT mice did not show changes in their capability for undergoing synaptic plasticity (Figure 1E). Thus, these data suggest that calpains are involved in the impairment of synaptic plasticity caused by overexpression of the APP and PS1 transgenes, and that treatment with a calpain inhibitor can rescue this impairment.

A problem frequently encountered when using calpain inhibitors is their specificity for calpains. E64 irreversibly modifies the active site cysteine of the protease (35-37). By contrast, BDA-410, $\left(\mathrm{C}_{26} \mathrm{H}_{32} \mathrm{~N}_{2} \mathrm{O}_{5} \mathrm{~S} ; \mathrm{MW}, 484.61\right.$; half-life, $\sim 400$ minutes), is a novel orally active synthetic Leu-Leu peptidomimetic with a cyclopropenone group that strongly draws toward itself the hydrogen of the $-\mathrm{SH}$ residues of cysteines contained in the calpain molecule (Figure 2A) (41). This leads to an intense electrostatic interaction between the resultant positively charged cyclopropenium ion and the $\mathrm{S}^{-}$residue in calpain cysteines. BDA- 410 has a potent and selective inhibitory action against calpain. Its inhibitory activities against specific proteases in SH-SY5Y cells were the following: calpain 1/calpain 2, $\mathrm{IC}_{50}=21.4 \mathrm{nM}$; papain, $\mathrm{IC}_{50}=400 \mathrm{nM}$; cathepsin B, $\mathrm{IC}_{50}=16 \mu \mathrm{M}$; thrombin, $\mathrm{IC}_{50}>100 \mu \mathrm{M}$; cathepsin $\mathrm{G}, \mathrm{IC}_{50}>100 \mu \mathrm{M}$; cathepsin D, $\mathrm{IC}_{50}=91.2 \mu \mathrm{M}$; proteasome 20S, $\mathrm{IC}_{50}>100 \mu \mathrm{M}(42)$.

To confirm the results with E64 using BDA-410, we first examined the effect of BDA-410 on basal mEPSC frequency in 11- to 14-day-old cultured neurons derived from APP/PS1 mice and WT littermates. We confirmed our previous findings that basal mEPSC frequency is increased in cultures from APP/PS1 mice compared with cultures from WT mice (Figure 2B). The average amplitude of mEPSCs was unchanged. In interleaved experiments, APP/PS1 cultures were treated with BDA-410 $(50 \mathrm{nM})$ (in 0.1\% DMSO). The application of BDA-410 to the culture medium for 3-4 days reestablished normal basal frequency of spontaneous neurotransmitter release in APP/PS1 mice (Figure 2B), while having no effect on the frequency of neurotransmitter release in WT cultures (Figure 2B). These results confirm the findings with E64, indicating that calpain inhibitors can be used to reestablish normal basal frequency of spontaneous release of neurotransmitter in APP/PS1 mice. Moreover, since the concentration of BDA-410 used in these experiments was very low (below the threshold of inactivation of other proteases), we have concluded that the effects of BDA-410 are specific to its inhibitory action against calpains. 

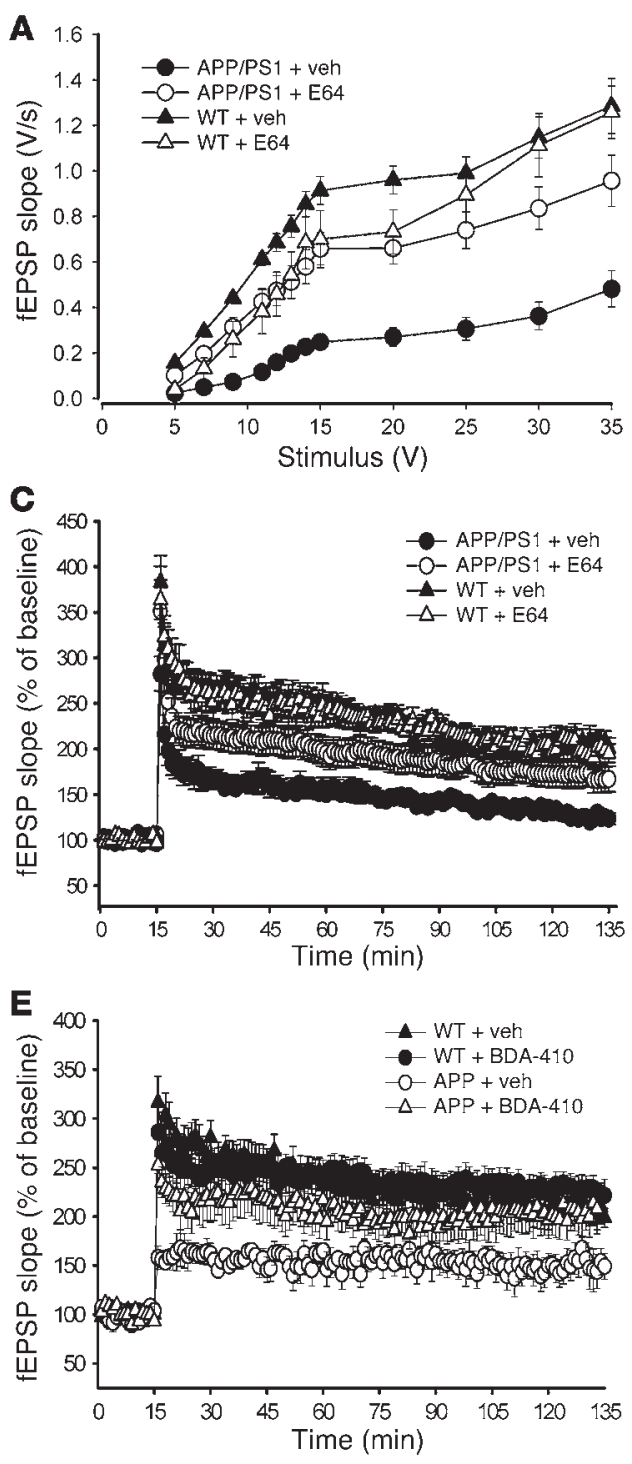

Figure 3

Calpain inhibition reestablished normal synaptic function following $A \beta$ elevation. (A) E64 improved BST at the CA3-CA1 connection of hippocampal slices from 7-month-old APP/PS1 mice treated for 5 months (10 slices from 9 E64-treated APP/PS1 mice, 7 slices from 7 vehicle-treated APP/PS1 mice, 8 slices from 8 vehicle-treated WT mice; $P<0.01$ comparing E64- and vehicle-treated APP/PS1 slices with 2-way ANOVA). BST was not affected in E64-treated WT animals ( 6 slices from 6 mice; $P>0.05$ ). (B) BDA-410 reestablished normal BST in APP/PS1 mice treated with inhibitor between 8 weeks and 7 months of age ( 7 slices from 7 BDA-410-treated APP/PS1 mice, 9 slices from 8 vehicle-treated APP/PS1 mice, 8 slices from 7 vehicle-treated WT mice; $P<0.01$ comparing BDA-410- and vehicle-treated APP/PS1 slices). BDA-410 did not affect BST in WT littermates (11 slices from 9 mice; $P>0.05$ ). (C) E64 was beneficial against LTP impairment at the same synapses as in A (vehicle-treated APP/PS1 mice versus vehicletreated WT mice, $P<0.01$ with 2-way ANOVA; E64-treated APP/PS1 mice versus vehicle-treated APP/ PS1 mice, $P<0.01)$. The inhibitor did not affect LTP in WT mice $(P>0.05)$. (D) BDA-410 reestablished normal LTP in APP/PS1 mice treated between 8 weeks and 7 months of age (BDA-410-treated APP/PS1 mice versus vehicle-treated APP/PS1 mice; $P<0.01$; same synapses as in B). BDA-410 did not affect LTP in WT littermates $(P>0.05)$. (E) BDA-410 reestablished normal LTP in APP mice treated between 8 and 11 to 12 months of age $(P<0.05$ in 6 slices from 6 BDA-410-treated APP mice versus 7 slices from 7 vehicle-treated APP mice; 7 slices from 7 vehicle-treated WT littermates). BDA-410 did not affect LTP in slices from WT littermates (7 slices from 7 mice; $P>0.05$ ). BST did not vary among the 4 groups of mice (data not shown). (F) BDA-410 reestablished normal LTP in A $\beta_{42}$-perfused slices ( 6 slices treated with BDA-410 plus $A \beta$ versus 8 A $\beta$-treated slices, $P<0.05$; 6 vehicle-treated slices versus 7 BDA-410-treated slices). The bar indicates the perfusion with $A \beta_{42}$.
To further confirm that BDA-410 can rescue changes of synaptic transmission in APP/PS1 mice, we examined the effects of the compound on glutamate-induced, longlasting enhancement of synaptic transmission in cultured hippocampal neurons. We reproduced our previous results, showing that a brief application of glutamate (200 $\mu \mathrm{M})$ did not produce enhancement of mEPSC frequency in cultures from APP/PS1 mice compared with cultures from WT mice (Figure 2C). A 50-nM BDA-410 exposure for 3-4 days prior to recording, however, reestablished normal synaptic plasticity in cultures from double transgenic animals (Figure 2C). BDA-410-treated WT mice did not show changes in their capability for synaptic plasticity (Figure 2C). Thus, these data confirm our results with E64 and indicate that calpains are involved in the impairment of synaptic plasticity caused by overexpression of APP and PS1 transgenes.

Calpain inbibition is beneficial against synaptic dysfunction following $A \beta$ elevation in adult mice. The results obtained with the culture system encouraged us to validate our hypothesis in adult double transgenic mice, which have a more mature cellular network and synapse pattern than cell cultures. In a first series of experiments, we confirmed that levels of the calpain-generated $150-\mathrm{kDa}$ fragment of spectrin were increased in hippocampi of adult APP/PS1 animals (Supplemental Figure 2, A and B). Then, we assessed whether E64 and BDA-410 inhibit calpain activity in mouse brains. We treated both WT and APP/PS1 mice with either E64 (6.4 mg/ $\mathrm{kg}$, i.p.), BDA-410 (30 mg/kg in $1 \%$ Tween 80 saline in a volume equal to $5 \mathrm{ml} / \mathrm{kg}$, per os), or vehicle for 60 days and then checked whether hippocampal calpain activity was affected. We found that the inhibitors 

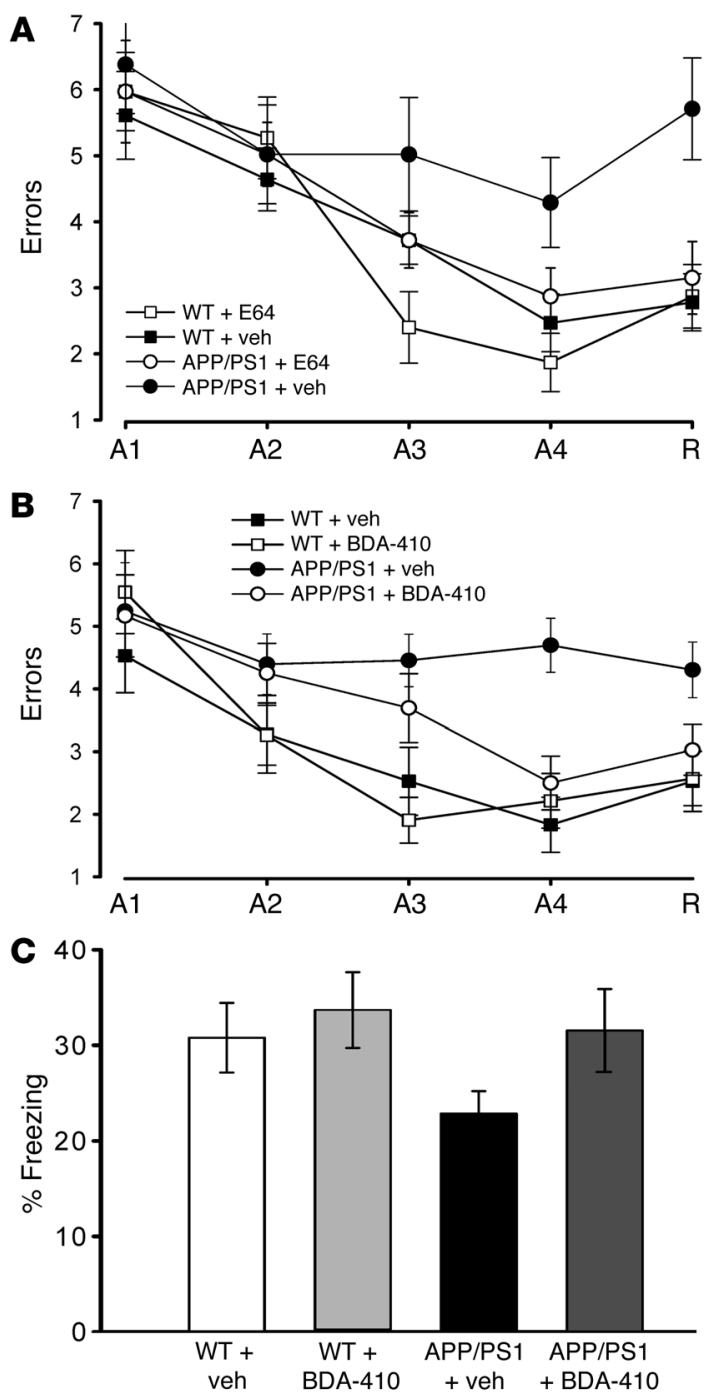

blocked the cleavage of spectrin, indicating that both E64 and BDA410 are able to inhibit calpain activity in the mouse brain (data not shown; $n=3$ animals for each group).

We next examined the function of the connection between Shaffer collateral and pyramidal neurons from CA1 stratum radiatum using extracellularly recorded field excitatory postsynaptic potentials (fEPSPs). Both APP/PS1 and WT mice were treated with E64, BDA-410, or vehicle from the age of 2 months (when plaques are just starting to appear) to 7 months (when plaque pathology is extensive) (38). As previously shown in 7-month-old double transgenics (38), we found that basal synaptic transmission (BST) was reduced in slices from vehicle-treated APP/PS1 mice compared with vehicle-treated WT littermate slices (Figure 3A). However, daily i.p. injections with E64 improved BST in APP/PS1 mice (Figure 3A). Moreover, treatment with vehicle did not affect BST in WT slices, as they showed similar values to control slices that did not receive any treatment (Supplemental Figure 3A). BST was not affected in E64treated WT animals (Figure 3A). Next, we replicated these findings with the more specific calpain inhibitor BDA-410. This treatment leads to a concentration of approximately $24 \mathrm{nM}$ in the plasma with a maximum concentration $\left(\mathrm{C}_{\max }\right)$ after per os bolus equal to $11.87 \mathrm{ng} / \mathrm{ml}$. BDA-410 improved BST in double transgenics with-

\section{Figure 4}

Calpain inhibition reestablished normal spatial-working memory and associative fear memory in APP/PS1 mice. (A) In RAWM testing APP/ PS1 mice treated with E64 for 5 months from 8 weeks of age made the same number of errors on the fourth acquisition (A4) and fifth retention trial $(\mathrm{R})$ as vehicle-treated WT mice $(n=13 ; P<0.05$ compared with vehicle-treated APP/PS1 mice with 2-way ANOVA; planned comparisons at trial A4, $P<0.05$, and trial $\mathrm{R}, P<0.01$ ), whereas vehicletreated double transgenics made more errors than vehicle-treated WT mice ( $n=7 ; P<0.01$ compared with vehicle-treated WT mice). E64 did not affect the performance of WT mice $(n=5 ; P>0.05$ compared with vehicle-treated WT mice). Visible platform task did not show any sensory-motor or motivational impairment in the same animals (data not shown). (B) BDA-410 reestablished normal spatial-working memory in APP/PS1 mice following 5 months of treatment from 8 weeks of age. BDA-410-treated double transgenics $(n=12)$ showed similar performance in the RAWM test as vehicle-treated WT littermates $(n=12)$, whereas vehicle-treated double transgenic mice $(n=11)$ showed abnormal learning and memory $(P<0.05$ in BDA-410-treated APP/PS1 mice compared with vehicle-treated APP/PS1 mice; planned comparisons showed that the 2 groups were significantly different at trial A4, $P<0.05$, and trial $R, P<0.01)$. The inhibitor did not affect the performance of WT mice $(n=14 ; P>0.05$ compared with vehicletreated WT mice). The visible platform task did not show any sensorymotor or motivational impairment in all groups (data not shown). (C) BDA-410 reestablished normal contextual learning in APP/PS1 mice following 5 months of treatment from 8 weeks of age $(P<0.01$ compared with vehicle-treated APP/PS1 mice). The inhibitor did not affect the performance of WT mice $(P>0.05)$. 
A
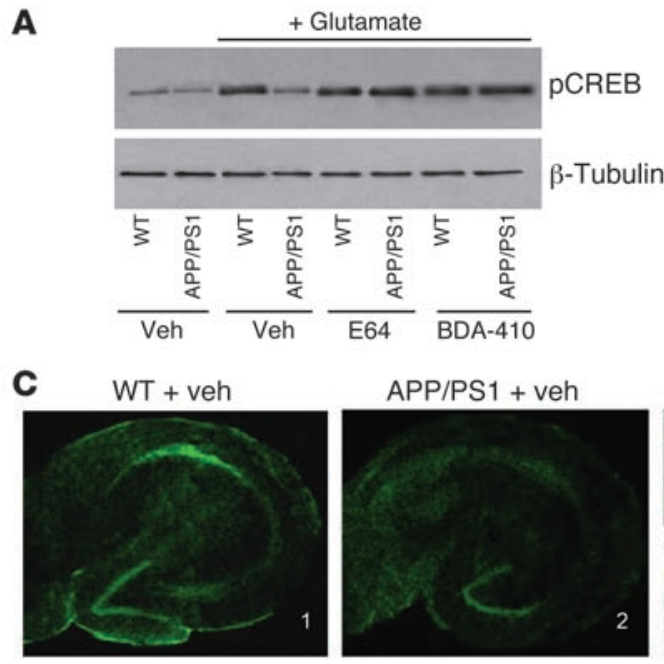

$\mathrm{APP} / \mathrm{PS} 1+\mathrm{E} 64$
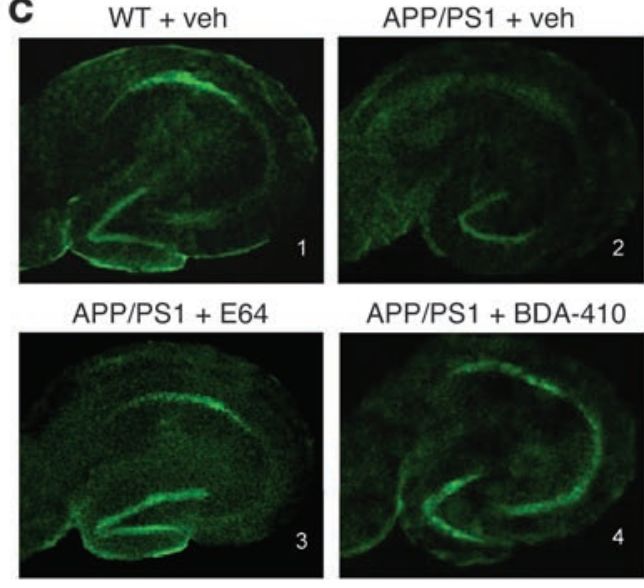

APP/PS1 + BDA-410
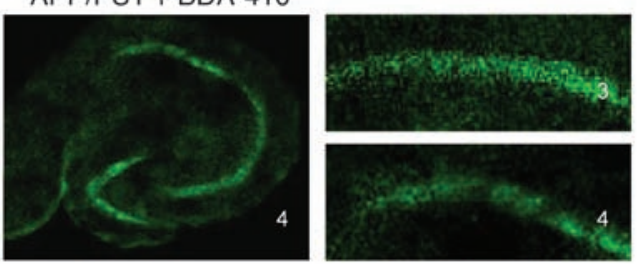

B
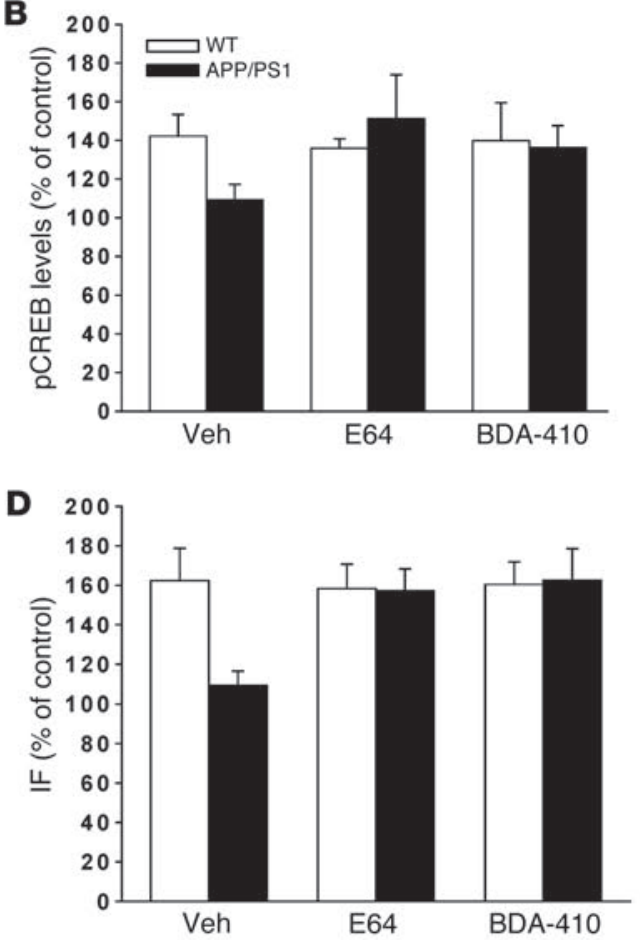

$\mathbf{F}$

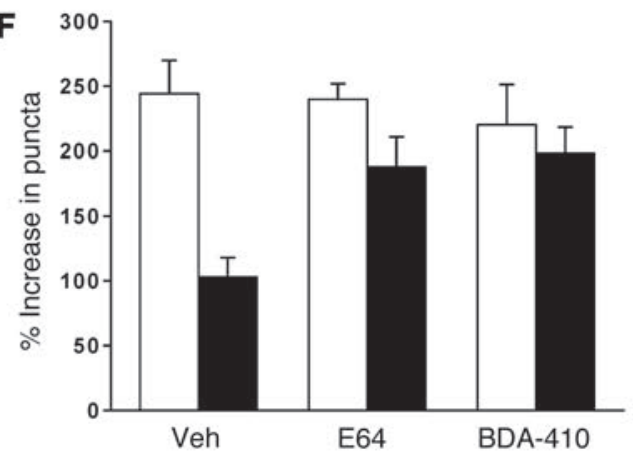

\section{Figure 5}

Calpain inhibition reestablished the increase in CREB phosphorylation during synaptic plasticity in APP/PS1 mice and produced a normal distribution of the synaptic protein synapsin I. (A) Western blot for pCREB at Ser-133 from cultured hippocampal neurons after 5-minute glutamate treatment. Five-day-old cultures were either treated with vehicle, E64, or BDA-410 for 3 days prior to applying glutamate. (B) Quantitative western blot analysis of data shown in A. pCREB levels in vehicle-treated WT cultures were increased after glutamate $(n=11 ; P<0.01)$. APP/PS1 cultures failed to present the pCREB increase $(n=7 ; P>0.01$ with 1 -way ANOVA). However, both E64 and BDA-410 reestablished normal pCREB values ( $n=5$ and 8 , respectively). E64 and BDA-410 did not affect phosphorylation in WT cultures ( $n=5$ and 7, respectively). All samples were normalized against $\alpha$-tubulin. (C) Examples of hippocampal slices stained with a pCREB antibody and fixed 60 minutes after tetanus in WT and APP/PS1 animals treated for 5 months from 8 weeks of age with E64, BDA-410, or vehicle. Lower-power (original magnification, $\times 4$ ) view of the entire slice (left and middle panels). Higher-power (original magnification, $\times 16$ ) view of CA1 cell pyramidal area (right panels). (D) Plot showing blockade of CA1-pCREB increase after tetanus in APP/PS1 slices ( $n=6$ both for WT and APP/PS1 slices; $P<0.01$ ), whereas treatment with both E64 and BDA-410 for 5 months from 8 weeks of age reestablished the tetanus-induced pCREB increase $(n=6$ for both; $P<0.01$ compared with tetanized slices from vehicletreated APP/PS1 mice). Both E64 and BDA-410 had no effect on WT mice after tetanus ( $n=6$ for both). In the absence of theta-burst, neither E64 nor BDA-410 induced changes in WT or APP/PS1 mice ( $n=4$ for each group; data not shown). IF, immunofluorescence. (E) The number of synapsin I-immunoreactive puncta was increased in vehicle-treated APP/PS1 cultures $(n=6)$ compared with vehicle-treated WT cultures $(n=6 ; P<0.01)$. However, the basal synapsin I-immunoreactive puncta number was normal after exposure to E64 $(n=7)$ or BDA-410 $(n=5)$. The inhibitors did not affect synapsin I immunoreactivity in WT cultures $(\mathrm{E} 64, n=6 ; \mathrm{BDA}-410, n=7$; $P<0.01$ for both compared with vehicle-treated APP/PS1 cultures). (F) Glutamate failed to increase synapsin I-immunoreactive puncta numbers in vehicle-treated APP/PS1 cultures $(n=8)$ compared with vehicle-treated WT cultures $(n=7, P<0.01)$, whereas both E64 and BDA-410 reestablished the glutamate-induced immunoreactivity increase in transgenic cultures (E64, $n=9$; BDA-410, $n=9$; $P<0.01$ for both compared with vehicle-treated APP/PS1 cultures). Both E64 and BDA-410 had no effect on glutamate-induced immunoreactivity increase in WT cultures (E64, $n=9$; BDA-410, $n=8$ ). 
3 months and found that the treatment reestablished normal LTP in 11- to 12-month-old APP mice compared with vehicle-treated APP littermates (Figure 3E). Thus, it is likely that this mutant form of APP plays a critical role in altering calpain activation and subsequently leading to changes in synaptic plasticity.

The APP and PS1 transgenes could affect neuronal function through a number of different mechanisms $(44,45)$, including direct effects by $A \beta$. The trafficking and signaling properties of the full-length form of APP and its natural cleavage products are likely different, which could impact aspects of synaptic function differently. To separate A $\beta$ effects from other effects of APP and PS1 overexpression, we determined whether A $\beta$ per se was responsible for the deficits observed in our studies on transgenic mice. We tested whether application of $\mathrm{A} \beta_{42}$ to WT slices was capable of reproducing the results obtained from transgenic mice. Since it has already been described that natural oligomers of human $A \beta$, in the absence of monomers and fibrils, markedly inhibit LTP in vivo (46), we applied 200-nM oligomeric $\mathrm{A} \beta_{42}$ concurrently with BDA-410 (100 nM) for 20 minutes to WT slices prior to inducing LTP. We confirmed that oligomeric $A \beta_{42}$ inhibited LTP and found that BDA-410 reestablished normal LTP in A $\beta$-treated slices (Figure $3 F$ ). Collectively, these experiments demonstrate that calpain inhibition can rescue the damage to synaptic plasticity caused by oligomeric forms of $\mathrm{A} \beta$.

Calpain inhibition is beneficial against cognitive loss in APP/PS1 mice. Plastic changes occurring at the synaptic level in the hippocampus are thought to underlie spatial learning. Memory loss is the most striking and well-known symptom of AD. Indeed, a major goal of a therapeutic agent against AD is to block or at least to alleviate memory decline. Thus, we ascertained whether treatment with calpain inhibitors could reverse or reduce the spatial learning impairment in APP/PS1 mice. In a series of experiments, we tested whether E64 is capable of reestablishing normal spatial memory in double transgenic animals. Both WT and APP/PS1 mice were i.p. injected with $6.4-\mathrm{mg} / \mathrm{kg}$ E64 or vehicle between 8 weeks and 7 months of age. At 7 months, APP/PS1 animals that had been injected with vehicle exhibited severe abnormalities in their spatial-working memory, as determined by radial-arm water maze (RAWM) testing (Figure 4A). Daily, i.p. injections of E64 improved the behavioral performance of the double transgenic mice (Figure 4A). Treatment with E64 did not affect the performance of WT mice (Figure 4A). Vehicle-treated WT mice had similar performance as control untreated WT mice (data not shown). We obtained similar results when we treated with BDA-410 $(30 \mathrm{mg} / \mathrm{kg}$ in $1 \%$ Tween 80 saline, in a volume equal to $5 \mathrm{ml} / \mathrm{kg}$, per os, treated daily from 8 weeks to 7 months of age; Figure 4B). To test for visual, motor, and motivational deficits, E64-, BDA-410-, and vehicletreated APP/PS1 mice and WT littermates, as well as untreated WT mice, underwent visible platform task after performing the RAWM test. We found no difference in speed and latency period to the platform for the various groups of mice (data not shown). These data indicate that calpain inhibition is capable of reestablishing normal spatial-working memory in APP/PS1 mice.

Another cognitive test that has been used in $\mathrm{AD}$ animal models is the fear-conditioning, associative learning paradigm. We first examined contextual fear learning, a hippocampus-dependent task, then we tested cued fear learning, a hippocampus-independent task. APP/PS1 and WT littermates treated with either vehicle or BDA-410 between the eighth week and the seventh month of age were subjected to a standard fear-conditioning paradigm. The mice had to associate a neutral stimulus with an aversive one. They were placed in a new context (fear-conditioning box) and exposed to a conditional stimulus (CS), i.e., a white noise cue, paired with an unconditional stimulus (US), i.e., a mild foot shock. Fear learning was assessed 24 hours later by measuring freezing behavior in response to presentation of the context (contextual conditioning) or of the auditory cue within a completely different context (cued conditioning). We found no difference in the freezing behavior of the vehicle-treated and BDA-410-treated APP/PS1 mice compared with vehicle-treated and BDA-410-treated WT littermates during the training phase of the fear-conditioning testing (data not shown). Twenty-four hours later we found decreased freezing behavior in vehicle-treated APP/PS1 mice compared with vehicle-treated WT littermates in the analysis of contextual learning (Figure 4C). However, treatment with BDA-410 reestablished normal freezing in APP/PS1 mice and did not affect the performance of WT mice (Figure 4C). Treatment with vehicle did not affect the performance of WT mice as they showed similar freezing time as control untreated WT mice (data not shown). We did not find a difference in freezing behavior during cued learning (data not shown). These results indicate that the impairment in contextual fear learning in APP/PS1 mice can be rescued by treatment with a calpain inhibitor.

Next, we examined whether the beneficial effects of calpain inhibition are through an action against the negative effects of APP overexpression. Similar to synaptic plasticity, we found that treatment with BDA-410 for 3 months was capable of reestablishing normal spatial-working memory and associative memory in 11- to 12-month-old APP mice (Supplemental Figure 4, A and B). Thus, it is likely that the expression of mutant APP plays a critical role in inducing calpain activation, leading to subsequent memory loss.

Calpain in bibition does not alter $A \beta$ levels. Given that $\mathrm{A} \beta$ elevation is a hallmark of $\mathrm{AD}$, we tested whether inhibiting calpain activity influences $A \beta$ levels. ELISA analysis of $A \beta_{40}$ and $A \beta_{42}$ revealed readily quantifiable levels of these peptides in APP/PS1 neuronal culture medium 10 days after plating (Supplemental Figure 5A). Medium of cultures from WT mice did not show detectable values for either $A \beta_{40}$ or $A \beta_{42}$. In interleaved experiments, we further observed that treating cultures from APP/PS1 mice for 3-4 days with E64 or BDA-410 had no effects on levels of $A \beta_{40}$ and $A \beta_{42}$ (Supplemental Figure 5A). As an additional test, we examined blood levels and formic acid-extractable brain levels of $A \beta_{40}$ and $A \beta_{42}$ (representing the soluble and insoluble pools of these peptides) following 5-month treatment with either E64, BDA-410, or vehicle in double transgenic mice and their WT littermates. At 7 months, vehicle-treated APP/PS1 mice showed high levels of A $\beta$ (Supplemental Figure 5, B and C), whereas WT littermates had undetectable levels. Daily treatment with E64 or BDA-410 between the eighth week and seventh month did not reduce brain or blood levels of $A \beta$ (Supplemental Figure 5, B and C). We also analyzed whether the inhibitors were capable of reducing plaque load. We did not find a reduction in the area occupied by plaques in either hippocampus or cortex by immunohistochemistry with the monoclonal antibody 4G8, which stains both fibrillar and diffuse A $\beta$ (Supplemental Figure 5D), or by staining with Congo red to analyze compact deposits (Supplemental Figure 5E). No A $\beta$ deposition (as assessed with either the 4G8 antibody or Congo red) was detected in WT mice. Collectively, these data support the hypothesis that calpain inhibitors enhance cognition without affecting amyloid levels.

The effect of calpain inhibition is mediated by CREB phosphorylation. Studies on cell cultures and slices after exogenous application of 
A

A $)$

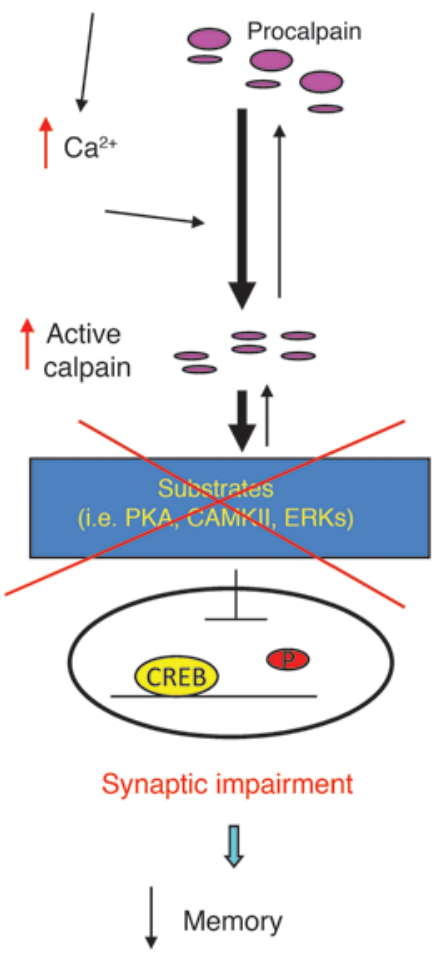

B
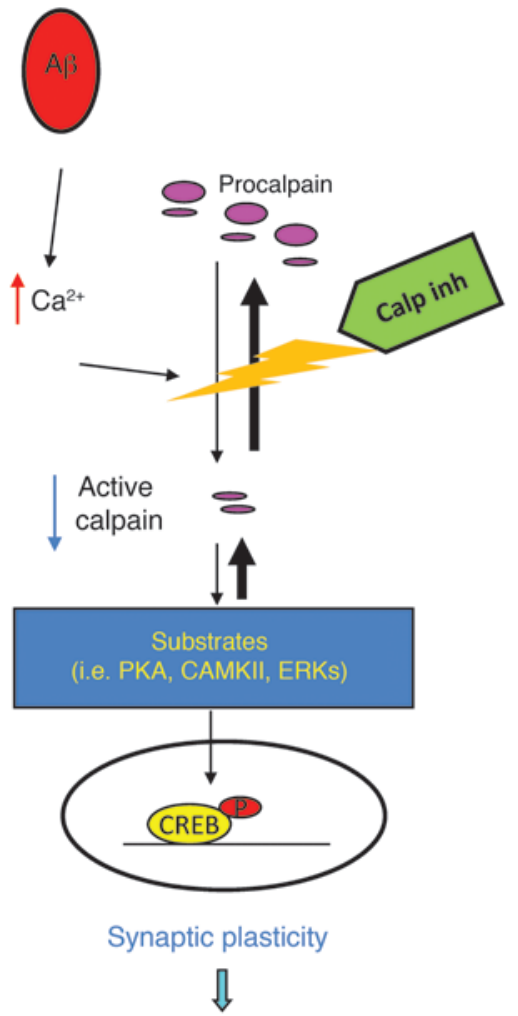

Memory
$A \beta_{42}$, as well as data on amyloid-depositing transgenic animal models, have shown a decrease in phosphorylation of a transcriptional mediator of synaptic plasticity and a key molecule in memory processes, the CREB protein $(19,20,47-49)$. Given that calpains might downregulate phosphorylation of CREB $(21,50)$, we investigated whether calpain inhibitors act on CREB phosphorylation during synaptic plasticity. We found that, unlike vehicle-treated or nontreated WT cultures (data not shown), both of which showed an increase in phosphorylated CREB (PCREB) in response to glutamate (200 $\mu \mathrm{M}, 5$ minutes), cultures from vehicle-treated APP/PS1 mice did not show this increase in CREB phosphorylation (Figure 5 , A and B). These results are consistent with previously reported findings using exogenous application of $A \beta$ (19). However, either E64 $(1 \mu \mathrm{M})$ or BDA-410 $(50 \mathrm{nM})$ applied daily for 3 days to 5 -dayold cultures reestablished the glutamate-induced increase in CREB phosphorylation in APP/PS1 cultures without affecting phosphorylation in WT cultures (Figure 5, A and B). Moreover, neither E64 nor BDA-410 affected basal pCREB levels in WT or APP/PS1 cultures (data not shown). Consistent with these findings, APP/ PS1 slices did not show the increase in PCREB induced by $\theta$ burst, as seen with an antibody specific to phosphoserine 133 in both vehicle- and non-treated WT slices. This inhibition was reversed in slices from mice that had been treated with either E64 or BDA-410 but not with vehicle (Figure 5, C and D). The inhibitors did not affect basal CREB phosphorylation in either WT or APP/PS1 slices (data not shown). Collectively, these data strongly support the possibility that calpain inhibitors act by reestablishing the increase in PCREB, thus rescuing the impairment of synaptic plasticity caused by overexpression of the APP and PS1 transgenes.

\section{Figure 6}

Working hypothesis on how calpain inhibitors (Calp-inh) may be able to improve synaptic dysfunction. (A) A $\beta$ activates a molecular cascade that leads to calpain activation and degradation of enzymes and structural proteins that are involved in learning and memory. Among the consequences of calpain activation, the transcription factor CREB cannot be phosphorylated and initiate transcription. (B) Calpain inhibition blocks the calcium-induced activation of calpain, preventing structural signaling alterations and allowing the phosphorylation of CREB.

Calpain inbibition restores distribution of the synaptic protein, synapsin I. Cell cultures permit visualization of changes in the distribution of synaptic protein during plasticity. Glutamate has been found to produce a long-lasting increase in the number of immunoreactive clusters, or puncta, of the synaptic protein synapsin I, as well as other synaptic proteins such as synaptophysin, $\alpha$-synuclein, GluR1, and PSD95, suggesting that a series of coordinated microstructural changes in protein distribution occurs at the synaptic level during plasticity, in which redistribution of synapsin I is an integral component (51, 52). Moreover, consistent with findings on changes of mEPSC frequency in APP/PS1 cultures, overexpression of the 2 transgenes has been associated with an increase in the basal number of synapsin Iimmunoreactive puncta, together with a block of the glutamate-induced increase in their number (32). Therefore, to further elucidate the beneficial effect of calpain inhibition at the synaptic level, we examined synapsin I immunoreactivity in APP/PS1 cultures in the presence of the calpain inhibitors, both in basal conditions and during synaptic plasticity. As previously described (32), we found an increase in the number of synapsin I-immunoreactive puncta in vehicle-treated APP/PS1 cultures compared with WT cultures either treated with vehicle (Figure 5E) or not treated (data not shown). Exposure to E64 $(1 \mu \mathrm{M})$ or BDA-410 $(50 \mathrm{nM})$ for 3-4 days reestablished the normal number of synapsin I-immunoreactive puncta in cultures from double transgenic mice (Figure 5E). Treatment with the inhibitors did not affect levels of synapsin I immunoreactivity in WT cultures (Figure 5E). Moreover, when we examined the glutamate-induced increase in the number of synapsin Iimmunoreactive puncta (52), we found that application of the excitatory amino acid $\left(200 \mu \mathrm{M}\right.$ in $0 \mathrm{Mg}^{2+}$ saline for approximately 1 minute) failed to increase the number of puncta in vehicle-treated APP/PS1 cultures, whereas it increased number of puncta in both E64- and BDA-410-treated APP/PS1 cultures as well as in vehicle-treated WT cultures (Figure 5F) and control cultures that did not receive any treatment (data not shown). The 2 inhibitors did not affect the glutamate-induced immunoreactivity increase in WT cultures (Figure 5F). Taken together, these findings suggest that calpain inhibition reestablishes normal distribution of synaptic protein both in basal conditions and during plasticity.

\section{Discussion}

In this study, we have demonstrated that the abnormal synaptic function and memory in APP/PS1 mice are largely reversed by either of 2 calpain inhibitors, E64 and BDA-410. To explain our 
results, we have proposed the working hypothesis illustrated in Figure 6. Upon binding with a putative membrane receptor, $A \beta$ activates a molecular cascade that leads to calpain activation and degradation of important proteins involved in synaptic plasticity and learning and memory. Among the consequences of this proteolysis, there is the decreased phosphorylation or increased degradation of the transcription factor CREB, resulting in a failure to maintain transcription, ultimately leading to synaptic dysfunction and cognitive abnormalities.

Calpains are at the crossroad between physiology and pathology $(2,53)$. Normal calpain activation plays a role in key signaling processes. Abnormal calpain activation, in turn, is responsible for the degradation of most of the cellular protein pool, including signaling proteins, transcription factors, and cytoskeletal substrates. Given that $\mathrm{Ca}^{2+}$ is elevated in AD brains (54), it is likely that loss of $\mathrm{Ca}^{2+}$ homeostasis disrupts physiological processes that regulate calpain activity in cells, leading to dysregulation of calpain activity. Consistent with this scenario, autolysis of calpain 1 to its 76- and $78-\mathrm{kDa}$ forms is enhanced in brains of AD patients (4), calpain 2 levels are increased in brains from $\operatorname{AD}$ patients $(5,55)$, and finally, levels of the endogenous inhibitor calpastatin are decreased (56).

The results obtained with E64 strongly support the hypotheses that inhibition of calpains might rescue changes of synaptic transmission and improve cognitive function in $\mathrm{AD}$ models. These observations are in agreement with data showing that the cysteine protease inhibitor leupeptin improves basal synaptic function in cell cultures from APP/PS1 mice (57). They are also in agreement with the finding that $\mathrm{A} \beta$-induced $\mathrm{Ca}^{2+}$ influx leads to the degradation of dynamin 1, a synaptic protein involved in endocytosis, via calpain activation (58). However, although E64 is a highly specific calpain inhibitor, the compound might have rescued changes of synaptic transmission and memory through inhibitory actions on other cysteine proteases. The consequence of such lack of specificity would have been the failure of BDA-410, a more powerful and specific calpain inhibitor, to improve synaptic transmission and cognition in the APP/PS1 mice. However, we have been able to demonstrate that BDA-410 is capable of improving basal neurotransmission, synaptic plasticity, as well as spatial-working memory and contextual learning following overexpression of mutated APP and PS1 transgenes. These findings are particularly relevant in light of the observations that synaptic alterations are highly correlated with the severity of clinical dementia, whereas other important pathologies in the disease, such as senile plaques and neurofibrillary tangles, are associated to a lesser extent (59).

Studies on AD mouse models and on slices exposed to sublethal levels of $A \beta_{42}$ have shown reduced levels of PCREB, a transcriptional mediator of LTP and memory $(19,20,47,49)$. Interestingly, analysis of the molecular mechanisms between aberrant APP metabolism and reductions of PCREB revealed an elevated calpain activity (21). Calpain inhibitors, in turn, reestablished normal pCREB levels (21). Consistent with these data, we have found that both E64 and BDA-410 reestablish normal levels of PCREB in a cell culture system and in slice preparations.

Another finding from our studies was the absence of changes in $A \beta$ levels following application of calpain inhibitors. This finding is consistent with our working hypothesis that places calpain activation downstream from $\mathrm{A} \beta$ binding to cellular targets and ultimately affects CREB phosphorylation (49). While not affecting total $A \beta$ levels, the inhibition of calpains may exert worthwhile therapeutic effects in $\mathrm{AD}$ by blocking the calpain-initiated cas- cades induced by both $\mathrm{A} \beta$ and other $\mathrm{AD}$ risk factors that converge on calpains. Nonetheless, it has been reported that calpains are involved in trafficking and, indirectly, processing of APP (7-11). Changes in a soluble pool of $A \beta(60)$, in aggregation state of nondeposited $A \beta$, or in the intraneuronal $A \beta$ pool $(40,61,62)$ that could not be detected because of the accelerated phenotype of the $\mathrm{APP} / \mathrm{PS} 1$ mice used in the present experiments might have also contributed to the beneficial effects of the 2 calpain inhibitors. Thus, the effect of both E64 and BDA-410 that we report here represents a potentially important mechanism for improving synaptic and memory deficits produced by $\mathrm{A} \beta$.

The physiological roles of calpains might be a source of concern, with drugs reducing calpain activity because inhibition might affect the physiological function of calpains, and cause adverse side effects. This seems less likely in view of studies on genetically modified mice overexpressing the endogenous inhibitor calpastatin, which show normal cytoskeletal components and synaptic markers as well as development, fertility, morphology, motility, and life span in normal conditions (63). Most tellingly, we saw no effect of E64 and BDA-410 on synaptic function, cognitive behavior, and locomotion in the WT mice used in our experiments. Indeed, calpains are likely to cleave polypeptides at a limited number of sites, leaving large, often catalytically active fragments (53). Thus, it is likely that calpains have a regulatory or signaling function in cells rather than a digestive function such as the lysosomal proteases, and therefore, undesired side effects by calpain inhibitors might be limited.

The results obtained with these studies support a new possible therapeutic strategy for $\mathrm{AD}$ based on modulating calpain actions. The calpain family of proteases constitutes a system that has not yet been extensively exploited in the therapy of $\mathrm{AD}$, although mounting evidence supports an early activation of calpains in vulnerable brain regions in $\operatorname{AD}(2,64)$. Since synaptic changes are highly correlated with the severity of clinical dementia (59), protecting against synaptic disruption through calpain inhibitors may be expected to stop or slow down memory impairment in AD and other neurodegenerative disorders characterized by abnormal amyloid production.

\section{Methods}

Animals. All experiments were performed with approval of the Columbia University Animal Care and Use Committee in accordance with the guidelines for the humane treatment of animals (NIH-Public Health Service Policy on Humane Care and Use of Laboratory Animals. Revised 2002). The animals used for generating cell cultures consisted of double transgenic mice expressing both the human APP mutation (K670N,M671L), and the human PS1 mutation (M146L) (line 6.2), as well as their WT littermates. They were obtained by crossing APP with PS1 animals. To identify the genotype of the animals, we used the polymerase chain reaction on samples of the tail taken after the dissection of the hippocampus $(57,65,66)$.

Cell cultures. Primary cultures were prepared from 1-day-old mouse pups (67). Cells were dissociated through enzymatic treatment ( $0.25 \%$ trypsin) and subsequent trituration. Hippocampal cells were grown in medium containing $84 \%$ MEM, supplemented with $10 \%$ heat-inactivated fetal calf serum, $45 \mathrm{mM}$ glucose, $1 \%$ MEM vitamin solution, and $2 \mathrm{mM}$ glutamine. After 24 hours, this medium was replaced by a medium containing $96.5 \%$ Neurobasal A, $2 \%$ B27-nutrient, $1 \%$ heat-inactivated fetal calf serum, $0.4 \mathrm{mM}$ glutamine, and $6.6 \mathrm{ng} / \mathrm{ml} 5$-fluorodioxyuridine in $16.4 \mathrm{ng} / \mathrm{ml}$ uridine to suppress cell division. To prevent the induction of plasticity by spontaneous synaptic activity, kynurenic acid $(0.5 \mathrm{mM})$ was also included in the culture medium. 
Immunocytochemistry. As previously described (51), cultures were incubated with 2 primary antibodies overnight at $4{ }^{\circ} \mathrm{C}$ : mouse monoclonal anti-calpain 1 (diluted 1:50; Chemicon International) and affinity-purified rabbit anti-synapsin I (diluted 1:200; Molecular Probes). The secondary antibodies were goat anti-rabbit labeled with Rhodamine Red and goat anti-mouse, labeled with Alexa Fluor 488 (diluted 1:500) in 4\% goat serum in PBS at room temperature for 1 hour.

Detection of CREB phosphorylation in hippocampal slices was performed as previously described (see Supplemental Methods) (20,68).

Western blot analysis. For assessment of calpain cleavage of spectrin in cell cultures, medium was removed and the attached cells were washed with TBS/EDTA twice. The cultured cells were subsequently harvested and homogenized in ice-cold buffer, consisting of $1 \mathrm{mM}$ EGTA, $2 \mathrm{mM}$ EDTA, $1 \mathrm{mM}$ DTT, $200 \mu \mathrm{g} / \mathrm{ml}$ leupeptin, $10 \mu \mathrm{g} / \mathrm{ml}$ aprotinin, $1 \mathrm{mM}$ PMSF, $2 \mu \mathrm{g} / \mathrm{ml}$ DNAase I, $20 \mathrm{mM}$ Tris-HCL, $\mathrm{pH}$ 7.4, and $150 \mathrm{mM} \mathrm{NaCl}$. Protein amounts of samples were determined by using the bicinchoninic acid (BCA) assay. Equal amounts of protein $(25 \mu \mathrm{g})$ were loaded on each lane of the SDS/PAGE gel (4\%-20\% [wt/vol] acrylamide), run with tris-tricine running buffer system, and transferred to PVDF membrane with a wet electrotransferring unit. The blots were probed for $\alpha$-tubulin as the internal standard control, stripped and reprobed with anti $\alpha$-spectrin $(1: 1,000)$ (MAB1622 nonerythroid; Chemicon), and membrane was exposed to alkaline phosphatase-conjugated anti-mouse $(1: 7,500)$ secondary antibodies. Detection was carried out using chemiluminescent substrate for alkaline phosphatase (CDP star) and exposure of the membrane to $\mathrm{x}$-ray film.

For assessment of calpain cleavage of spectrin on mice, the animals were perfused with PBS, and brains were dissected and homogenized in ice-cold buffer, consisting of $1 \%$ SDS, $1 \mathrm{mM}$ EGTA, $1 \mathrm{mM}$ DTT, $10 \mu \mathrm{g} / \mathrm{ml}$ aprotinin, $1 \mathrm{mM}$ PMSF, $2 \mu \mathrm{g} / \mathrm{ml}$ DNAase I, $20 \mathrm{mM}$ Tris-HCL, $\mathrm{pH}$ 7.4, and 150 $\mathrm{mM} \mathrm{NaCl}$. A concern with these experiments is that calpains are hyperactivated during the removal of the brain or after its removal for abnormal elevation of calcium levels. To avoid these problems, calpain activity was rapidly blocked by adding the calpain inhibitor, leupeptin $(200 \mu \mathrm{g} / \mathrm{ml})$, and by chelating calcium with EDTA $(5 \mathrm{mM})$, before homogenizing the tissue. The samples were centrifuged at $18,000 \mathrm{~g}$ for 30 minutes. Protein concentrations were determined by BCA protein assay kit (Pierce). Equal amounts of protein $(15 \mu \mathrm{g})$ were loaded on each lane of the SDS/PAGE gel (4\%-15\% gradient), run with tris-glycine running buffer system, and transferred to nitrocellulose membrane. The blots were probed for $\alpha$-tubulin as the internal standard control, stripped and reprobed with anti- $\alpha$-spectrin $(1: 1,000)$ (MAB1622; Chemicon), and membrane was exposed to alkaline phosphatase-conjugated anti-mouse $(1: 12,500)$ secondary antibodies. Detection was carried out using chemiluminescent substrate for alkaline phosphatase (CDP star) and exposure of the membrane to $\mathrm{x}$-ray film.

For assessment of PCREB in cultures, cultures were washed twice with normal saline bath solution. Cells were exposed to glutamate $(200 \mu \mathrm{M})$ in $\mathrm{Mg}^{2+}$ free bath solution for 5 minutes. After 20 minutes, cells were harvested and homogenized in $100 \mu \mathrm{l}$ of ice-cold buffer, consisting of $25 \mathrm{mM}$ sodium phosphate buffer, $1 \%$ SDS, $5 \mathrm{mM}$ EDTA, and $1 \mathrm{mM}$ PMSF. Protein concentrations were determined by BCA protein assay kit (Pierce), and equal amounts of protein $(15 \mu \mathrm{g})$ were loaded on the $4 \%-20 \%$ SDS/ PAGE gel (Invitrogen). The blots were probed for rabbit polyclonal antipCREB (1:1,000; Upstate Biotech.) and mouse monoclonal anti- $\beta$-tubulin (1:500; Sigma-Aldrich). Detection was performed using HRP-conjugated secondary antibodies (Pierce).

Electrophysiology. Cultured neurons were voltage clamped with the whole cell ruptured patch technique throughout the experiment. The bath solution consisted of the following: $119 \mathrm{mM} \mathrm{NaCl}, 5 \mathrm{mM} \mathrm{KCl}, 20 \mathrm{mM}$ HEPES, $2 \mathrm{mM} \mathrm{CaCl}_{2}, 2 \mathrm{mM} \mathrm{MgCl}_{2}, 30 \mathrm{mM}$ glucose, $0.001 \mathrm{mM}$ glycine, and picrotoxin, $0.1 \mathrm{mM}, \mathrm{pH} 7.3$, and osmolarity was adjusted to $330 \mathrm{mOsm}$ with sucrose.
The solution in the whole cell patch electrode consisted of the following: $130 \mathrm{mM}$ K-gluconate, $10 \mathrm{mM} \mathrm{KCl}, 5 \mathrm{mM} \mathrm{MgCl}$, $0.6 \mathrm{mM}$ EGTA, $5 \mathrm{mM}$ HEPES, $0.06 \mathrm{mM} \mathrm{CaCl}_{2}$, $2 \mathrm{mM}$ Mg-ATP, $0.2 \mathrm{mM}$ GTP, $0.2 \mathrm{mM}$ leupeptin, $20 \mathrm{mM}$ phosphocreatine, and $50 \mathrm{U} / \mathrm{ml}$ creatine-phosphokinase. Currents were recorded with a Warner amplifier (model PC-501A) and filtered at $1 \mathrm{kHz}$. In order to eliminate artifacts due to variation of the seal properties, the access resistance was monitored for constancy throughout all experiments. In order to suppress action potentials, $1 \mu \mathrm{M}$ tetrodotoxin was added to the bath when recording mEPSCs. They were digitized and analyzed with the mini analysis program (version 4.0) from Synaptosoft Inc.

Slice recordings were performed as described previously (49). Briefly, 400- $\mu \mathrm{m}$ slices were cut with a tissue chopper and maintained in an interface chamber at $29^{\circ} \mathrm{C}$ for 90 minutes prior to recording. CA1 fEPSPs were recorded by placing both the stimulating and the recording electrodes in CA1 stratum radiatum. BST was assayed either by plotting the stimulus voltages against slopes of fEPSP or by plotting the peak amplitude of the fiber volley against the slope of the fEPSP. For LTP experiments, a 15 -minute baseline was recorded every minute at an intensity that evokes a response approximately $35 \%$ of the maximum evoked response. LTP was induced using $\theta$-burst stimulation (4 pulses at $100 \mathrm{~Hz}$, with the bursts repeated at $5 \mathrm{~Hz}$, and each tetanus including 3 ten-burst trains separated by 15 seconds).

$A \beta$ preparation. Oligomeric $A \beta_{42}$ was prepared as previously described (20). Briefly, the lyophilized peptide (American Peptide) was resuspended in 100\% 1,1,1,3,3,3-hexafluoro-2-propanol (HFIP; Sigma-Aldrich), and HFIP was allowed to evaporate in a fume hood. The resulting clear peptide films were stored at $-20^{\circ} \mathrm{C}$. Twenty-four hours prior to use, the aliquots were added to DMSO (Sigma-Aldrich) and sonicated for 10 minutes. Oligomeric $A \beta_{42}$ was obtained by diluting an aliquot of $A \beta_{42}-$ DMSO solution with a small volume of normal recording bath solution, vortexing for 30 seconds, and then incubating at $4^{\circ} \mathrm{C}$ for 16 hours. This synthetic $\mathrm{A} \beta$ has been characterized both biochemically and electrophysiologically, showing similar biological effects at low nanomolar concentrations as naturally secreted oligomers of $A \beta$ (69). In addition, its normal sequence - but not a scrambled sequence - is known to block LTP immediately and consistently (20).

Behavioral studies. Assessments of both spatial-working memory through the RAWM and associative memory through analysis of contextual and cued conditioning have been previously described (see Supplemental Methods) (49).

A $\beta$ measurement. For cell cultures, supernatant derived from the medium collected from the culture dishes were spun at $2,500 \mathrm{~g}$ for 5 minutes at $4{ }^{\circ} \mathrm{C}$. For adult mice, hemibrains were frozen at $-80^{\circ} \mathrm{C}$, homogenized in sucrose buffer, and extracted in formic acid as previously described (70). Blood was harvested in a tube containing $10 \mathrm{mM}$ EDTA, centrifuged at $2,000 \mathrm{~g}$ for 5 minutes at $4^{\circ} \mathrm{C}$, and plasma was stored at $-80^{\circ} \mathrm{C}$ before loading onto ELISA plates. Human A $\beta$ levels were measured through ELISA, in which $A \beta$ was trapped with either a monoclonal antibody specific for $A \beta_{40}$ $\left(\mathrm{JRF} / \mathrm{cA} \beta_{40} / 10\right)$ or one specific for $\mathrm{A} \beta_{42}\left(\mathrm{JRF} / \mathrm{cA} \beta_{42} / 26\right)$, and then detected with a monoclonal antibody specific for whole $A \beta\left(J R F / A \beta_{\text {tot }} / 17\right)$ conjugated to horseradish peroxidase as previously described (71).

Plaque measurement. The method for evaluation of plaque load has been previously described (38). Briefly, immunostaining for plaques containing both fibrillar and diffuse $A \beta$ was performed with monoclonal 4G8 antibodies (Signet Laboratories). The specificity of immunoreactivity was confirmed by checking that no signal was detected when the primary antibody was omitted. Secondary antibodies consisted of biotinylated antimouse IgG. Analysis of immunoreactive deposits for $A \beta$ was done using the public domain program NIH image (version 1.61; http://rsb.info.nih. gov/nih-image/index.html), by defining a region of interest and setting a 
threshold to discriminate nonspecific staining. The percentage of surface area covered by $\mathrm{A} \beta$ immunoreactivity was used to measure plaque load. Compact deposits were measured through Congo red staining (Accustain Amyloid Staining kit; Sigma-Aldrich). Sections were incubated in an alkaline sodium chloride solution for 20 minutes and incubated in alkaline Congo red solution. Congo red staining was evaluated with a light microscope. The percentage of surface area that was stained with Congo red was used to measure Congo red-positive deposits with NIH image analysis software (v. 1.61; http://rsb.info.nih.gov/nih-image/index.html). Both for 4G8 staining and Congo red staining, 2 regions were analyzed: an area of parietal cortex, comprising layers I-VI, and an area of the hippocampal formation, including stratum oriens, pyramidal layer, stratum radiatum, and the dentate gyrus.

Statistics. Statistical analyses were performed blind with respect to genotype and treatment. Results were analyzed with 2-tailed Student's $t$ test (pairwise comparisons) or 2-way ANOVA with post-hoc correction (multiple comparisons). Results were expressed as mean \pm SEM. The level of significance was set for $P<0.05$.

1. Huang, Y., and Wang, K.K. 2001. The calpain family and human disease. Trends Mol. Med. 7:355-362.

2. Nixon, R.A. 2003. The calpains in aging and agingrelated diseases. Ageing Res. Rev. 2:407-418.

3. Perlmutter, L.S., et al. 1988. The ultrastructural localization of calcium-activated protease "calpain" in rat brain. Synapse. 2:79-88.

4. Saito, K., Elce, J.S., Hamos, J.E., and Nixon, R.A. 1993. Widespread activation of calcium-activated neutral proteinase (calpain) in the brain in Alzheimer disease: a potential molecular basis for neuronal degeneration. Proc. Natl. Acad. Sci. U. S. A. 90:2628-2632.

5. Grynspan, F., Griffin, W.R., Cataldo, A., Katayama, S., and Nixon, R.A. 1997. Active site-directed antibodies identify calpain II as an early-appearing and pervasive component of neurofibrillary pathology in Alzheimer's disease. Brain Res. 763:145-158.

6. Wang, K.K. 2000. Calpain and caspase: can you tell the difference? Trends Neurosci. 23:20-26.

7. Siman, R., Card, J.P., and Davis, L.G. 1990. Proteolytic processing of beta-amyloid precursor by calpain I. J. Neurosci. 10:2400-2411.

8. Chen, M., Durr, J., and Fernandez, H.L. 2000. Possible role of calpain in normal processing of betaamyloid precursor protein in human platelets. Biochem. Biophys. Res. Commun. 273:170-175.

9. Mathews, P.M., et al. 2002. Calpain activity regulates the cell surface distribution of amyloid precursor protein. Inhibition of clapains enhances endosomal generation of beta-cleaved C-terminal APP fragments. J. Biol. Chem. 277:36415-36424.

10. Steiner, H., et al. 1998. Expression of Alzheimer's disease-associated presenilin- 1 is controlled by proteolytic degradation and complex formation. J. Biol. Chem. 273:32322-32331.

11. Zhang, L., Song, L., and Parker, E.M. 1999. Calpain inhibitor I increases beta-amyloid peptide production by inhibiting the degradation of the substrate of gamma-secretase. Evidence that substrate availability limits beta-amyloid peptide production. J. Biol. Chem. 274:8966-8972.

12. Pontremoli, S., et al. 1987. Phosphorylation and proteolytic modification of specific cytoskeletal proteins in human neutrophils stimulated by phorbol 12-myristate 13-acetate. Proc. Natl. Acad. Sci. U. S. A. 84:3604-3608.

13. Gandy, S., Czernik, A.J., and Greengard, P. 1988. Phosphorylation of Alzheimer disease amyloid precursor peptide by protein kinase $\mathrm{C}$ and $\mathrm{Ca} 2+$ / calmodulin-dependent protein kinase II. Proc. Natl. Acad. Sci.U. S. A. 85:6218-6221.

14. Wang, K.K., and Yuen, P.W. 1997. Development and therapeutic potential of calpain inhibitors. $A d v$.

\section{Acknowledgments}

We thank Karen Hsiao-Ashe (University of Minnesota and The Mayo Clinic) for providing the APP mice, Karen Duff (Columbia University) for providing the PS1 mice, and Archana Narasanna and Fortunato Battaglia for assistance with western blot analysis and electrophysiological experiments, respectively. This work was supported by NIH National Institute of Neurological Disorders and Stroke (NINDS) (NS049442) and Institute for the Study of Aging (ISOA).

Received for publication October 17, 2007, and accepted in revised form May 14, 2008.

Address correspondence to: Ottavio Arancio, Department of Pathology and Taub Institute for Research on Alzheimer's Disease and the Aging Brain, Columbia University, P\&S 12-420D, $630 \mathrm{~W}$ 168th Street, New York, New York 10032, USA. Phone: (212) 8514617; Fax: (212) 851-4602; E-mail: oa1@columbia.edu.

Pharmacol. 37:117-152.

15. Banno, Y., Nakashima, S., Hachiya, T., and Nozawa, Y. 1995. Endogenous cleavage of phospholipase Cbeta 3 by agonist-induced activation of calpain in human platelets. J. Biol. Chem. 270:4318-4324.

16. Lee, M.S., et al. 2000. Neurotoxicity induces cleavage of p35 to p25 by calpain. Nature. 405:360-364.

17. Carillo, S., et al. 1994. Differential sensitivity of FOS and JUN family members to calpains. Oncogene. 9:1679-1689.

18. Lin, Y.C., Brown, K., and Siebenlist, U. 1995. Activation of NF-kappa B requires proteolysis of the inhibitor I kappa B-alpha: signal-induced phosphorylation of I kappa B-alpha alone does not release active NF-kappa B. Proc. Natl. Acad. Sci.U. S. A. 92:552-556.

19. Vitolo, O.V., et al. 2002. Amyloid beta -peptide inhibition of the PKA/CREB pathway and long-term potentiation: reversibility by drugs that enhance cAMP signaling. Proc. Natl. Acad. Sci. U. S. A. 99:13217-13221.

20. Puzzo, D., et al. 2005. Amyloid-beta peptide inhibits activation of the nitric oxide/cGMP/cAMPresponsive element-binding protein pathway during hippocampal synaptic plasticity. J. Neurosci. 25:6887-6897.

21. Mbebi, C., et al. 2002. Amyloid precursor protein family-induced neuronal death is mediated by impairment of the neuroprotective calcium/ calmodulin protein kinase IV-dependent signaling pathway. J. Biol. Chem. 277:20979-20990.

22. Siman, R., Baudry, M., and Lynch, G. 1984. Brain fodrin: substrate for calpain I, an endogenous calcium-activated protease. Proc. Natl. Acad. Sci. U. S. A. 81:3572-3576.

23. Johnson, G.V., Litersky, J.M., and Jope, R.S. 1991. Degradation of microtubule-associated protein 2 and brain spectrin by calpain: a comparative study. J. Neurochem. 56:1630-1638.

24. Yuen, E.Y., Liu, W., and Yan, Z. 2007. The phosphorylation state of GluR1 subunits determines the susceptibility of AMPA receptors to calpain cleavage. J. Biol. Chem. 282:16434-16440.

25. Wu, Y., et al. 2007. Truncations of amphiphysin I by calpain inhibit vesicle endocytosis during neural hyperexcitation. EMBO J. 26:2981-2990.

26. Shimizu, K., Phan, T., Mansuy, I.M., and Storm, D.R. 2007. Proteolytic degradation of SCOP in the hippocampus contributes to activation of MAP kinase and memory. Cell. 128:1219-1229.

27. Sant'Angelo, A., Trinchese, F., and Arancio, O. 2003. Usefulness of behavioral and electrophysiological studies in transgenic models of Alzheimer's disease. Neurochem. Res. 28:1009-1015.
28. Bliss, T.V., and Collingridge, G.L. 1993. A synaptic model of memory: long-term potentiation in the hippocampus. Nature. 361:31-39.

29. Arancio, O., Kandel, E.R., and Hawkins, R.D. 1995. Activity-dependent long-term enhancement of transmitter release by presynaptic $3^{\prime}, 5^{\prime}$-cyclic GMP in cultured hippocampal neurons. Nature. 376:74-80.

30. Malgaroli, A., and Tsien, R.W. 1992. Glutamateinduced long-term potentiation of the frequency of miniature synaptic currents in cultured hippocampal neurons. Nature. 357:134-139.

31. Arancio, O., et al. 1996. Nitric oxide acts directly in the presynaptic neuron to produce long-term potentiation in cultured hippocampal neurons. Cell. 87:1025-1035.

32. Trinchese, F., et al. 2004. Cell cultures from animal models of Alzheimer's disease as a tool for faster screening and testing of drug efficacy. J. Mol. Neurosci. 24:15-21.

33. Takahashi, R.H., et al. 2004. Oligomerization of Alzheimer's beta-amyloid within processes and synapses of cultured neurons and brain. J. Neurosci. 24:3592-3599.

34. Holcomb, L., et al. 1998. Accelerated Alzheimertype phenotype in transgenic mice carrying both mutant amyloid precursor protein and presenilin 1 transgenes. Nat. Med. 4:97-100.

35. Wang, K.K.W., and Yuen, P.-W. 1999. Calpain: pharmacology and toxicology of a calcium-dependent protease. K.K.W. Wang and P.-W. Yuen, editors. CRC Press/ Taylor \& Francis Group. Philadephia, Pennsylvania, USA. 448 pp.

36. Hashida, S., Towatari, T., Kominami, E., and Katunuma, N. 1980. Inhibitions by E-64 derivatives of rat liver cathepsin B and cathepsin $L$ in vitro and in vivo. J. Biochem. 88:1805-1811.

37. Chard, P.S., Bleakman, D., Savidge, J.R., and Miller, R.J. 1995. Capsaicin-induced neurotoxicity in cultured dorsal root ganglion neurons: involvement of calcium-activated proteases. Neuroscience. 65:1099-1108.

38. Trinchese, F., et al. 2004. Progressive age-related development of Alzheimer-like pathology in APP/ PS1 mice. Ann. Neurol. 55:801-814.

39. Chapman, P.F., et al. 1999. Impaired synaptic plasticity and learning in aged amyloid precursor protein transgenic mice. Nat. Neurosci. 2:271-276.

40. Oddo, S., et al. 2003. Triple-transgenic model of Alzheimer's disease with plaques and tangles: intracellular Abeta and synaptic dysfunction. Neuron. 39:409-421.

41. Li, X., Chen, H., Jeong, J.J., and Chishti, A.H. 2007. BDA-410: a novel synthetic calpain inhibitor active 
against blood stage malaria. Mol. Biochem. Parasitol. 155:26-32.

42. Yoshii, N., et al. 1999. Neuroprotective effects of a novel orally active reversible calpain inhibitor BDA410 [abstract]. Presented at the Society for Neuroscience 28th Annual Meeting. October 23-28. Miami Beach, Florida, USA. 344.

43. Chan, S.L., Culmsee, C., Haughey, N., Klapper, W., and Mattson, M.P. 2002. Presenilin-1 mutations sensitize neurons to DNA damage-induced death by a mechanism involving perturbed calcium homeostasis and activation of calpains and caspase-12. Neurobiol. Dis. 11:2-19.

44. Cao, X., and Sudhof, T.C. 2001. A transcriptionally [correction of transcriptively] active complex of APP with Fe65 and histone acetyltransferase Tip60. Science. 293:115-120.

45. Kamal, A., Almenar-Queralt, A., LeBlanc, J.F., Roberts, E.A., and Goldstein, L.S. 2001. Kinesin-mediated axonal transport of a membrane compartment containing beta-secretase and presenilin- 1 requires APP. Nature. 414:643-648.

46. Walsh, D.M., et al. 2002. Naturally secreted oligomers of amyloid beta protein potently inhibit hippocampal long-term potentiation in vivo. Nature. 416:535-539.

47. Dineley, K.T., et al. 2001. Beta-amyloid activates the mitogen-activated protein kinase cascade via hippocampal alpha7 nicotinic acetylcholine receptors: In vitro and in vivo mechanisms related to Alzheimer's disease. J. Neurosci. 21:4125-4133.

48. Dineley, K.T., Xia, X., Bui, D., Sweatt, J.D., and Zheng, H. 2002. Accelerated plaque accumulation, associative learning deficits, and up-regulation of alpha 7 nicotinic receptor protein in transgenic mice coexpressing mutant human presenilin 1 and amyloid precursor proteins. J. Biol. Chem. 277:22768-22780.

49. Gong, B., et al. 2004. Persistent improvement in synaptic and cognitive functions in an Alzheimer mouse model following rolipram treatment. J. Clin. Invest. 114:1624-1634.

50. Silva, A.J., Kogan, J.H., Frankland, P.W., and Kida, S. 1998. CREB and memory. Annu. Rev. Neurosci. 21:127-148.

51. Liu, S., et al. 2004. alpha-Synuclein produces a long-lasting increase in neurotransmitter release. EMBO J. 23:4506-4516.

52. Antonova, I., et al. 2001. Rapid increase in clusters of presynaptic proteins at onset of long-lasting potentiation. Science. 294:1547-1550.

53. Goll, D.E., Thompson, V.F., Li, H., Wei, W., and Cong, J. 2003. The calpain system. Physiol. Rev. 83:731-801.

54. Leissring, M.A., et al. 2000. Capacitative calcium entry deficits and elevated luminal calcium content in mutant presenilin-1 knockin mice. J. Cell Biol. 149:793-798.

55. Tsuji, T., Shimohama, S., Kimura, J., and Shimizu, K. 1998. m-Calpain (calcium-activated neutral proteinase) in Alzheimer's disease brains. Neurosci. Lett. 248:109-112.

56. Nixon, R.A. 2004. Niemann-Pick Type C disease and Alzheimer's disease: the APP-endosome connection fattens up. Am. J. Pathol. 164:757-761.

57. Di Rosa, G., Odrijin, T., Nixon, R.A., and Arancio, O. 2002. Calpain inhibitors: a treatment for Alzheimer's disease. J. Mol. Neurosci. 19:135-141.

58. Kelly, B.L., and Ferreira, A. 2006. beta-Amyloidinduced dynamin 1 degradation is mediated by $\mathrm{N}$-methyl-D-aspartate receptors in hippocampal neurons. J. Biol. Chem. 281:28079-28089.

59. Masliah, E. 1995. Mechanisms of synaptic dysfunction in Alzheimer's disease. Histol. Histopathol. 10:509-519.

60. Morgan, D., et al. 2000. A beta peptide vaccination prevents memory loss in an animal model of Alzheimer's disease. Nature. 408:982-985.

61. LaFerla, F.M., Troncoso, J.C., Strickland, D.K.,
Kawas, C.H., and Jay, G. 1997. Neuronal cell death in Alzheimer's disease correlates with apoE uptake and intracellular Abeta stabilization. J. Clin. Invest. 100:310-320.

62. Gouras, G.K., et al. 2000. Intraneuronal Abeta 42 accumulation in human brain. Am. J. Pathol. 156:15-20.

63. Higuchi, M., et al. 2005. Distinct mechanistic roles of calpain and caspase activation in neurodegeneration as revealed in mice overexpressing their specific inhibitors. J. Biol. Chem. 280:15229-15237.

64. Veeranna, et al. 2004. Calpain mediates calciuminduced activation of the erk1,2 MAPK pathway and cytoskeletal phosphorylation in neurons: relevance to Alzheimer's disease. Am. J. Pathol. 165:795-805.

65. Duff, K., et al. 1996. Increased amyloid-beta42(43) in brains of mice expressing mutant presenilin 1 . Nature. 383:710-713.

66. Hsiao, K., et al. 1996. Correlative memory deficits, Abeta elevation, and amyloid plaques in transgenic mice. Science. 274:99-102.

67. Ninan, I., and Arancio, O. 2004. Presynaptic CaMKII is necessary for synaptic plasticity in cultured hippocampal neurons. Neuron. 42:129-141.

68. Gong, B., et al. 2006. Ubiquitin hydrolase UchL1 rescues beta-amyloid-induced decreases in synaptic function and contextual memory. Cell. 126:775-788

69. Haass, C., and Selkoe, D.J. 2007. Soluble protein oligomers in neurodegeneration: lessons from the Alzheimer's amyloid beta-peptide. Nat. Rev. Mol. Cell. Biol. 8:101-112.

70. Schmidt, S.D., Jiang, Y., Nixon, R.A., Mathews, P.M. 2005. Tissue processing prior to protein analysis and amyloid-beta quantitation. Methods Mol. Biol. 299:267-278

71. Schmidt, S.D., Nixon, R.A., and Mathews, P.M 2005. ELISA method for measurement of amyloidbeta levels. Methods Mol. Biol. 299:279-297. 